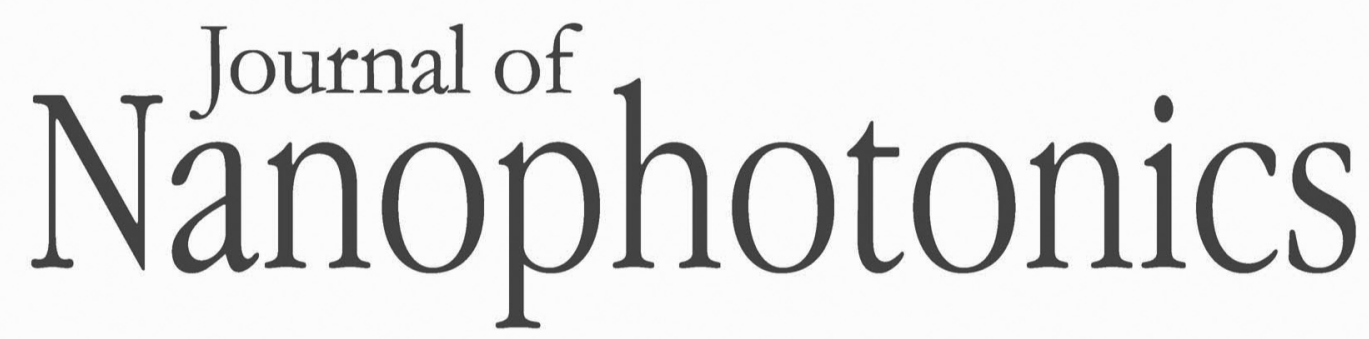

SPIEDigitalLibrary.org/jnp

\title{
Second Harmonic Generation in Plasmonic Nanoresonators
}

Marco Centini

Alessio Benedetti 


\title{
Second Harmonic Generation in Plasmonic Nanoresonators
}

\author{
Marco Centini and Alessio Benedetti \\ Sapienza Università di Roma, Dipartimento di Scienze di Base e Applicate per l'Ingegneria, \\ Via A. Scarpa 16, 00161, Roma \\ marco.centini@uniroma1.it
}

Paper 12171CT received Nov. 15, 2012; revised manuscript received Dec. 2, 2012; accepted for publication Dec. 3, 2012; published online Jan. 21, 2013.

Nonlinear effects can be enhanced and tailored on a subwavelength scale by taking advantage of high field confinement by selective coupling among resonant plasmonic nanostructures, called plasmonic nanoresonators and nanoantennas. In particular, we focus our attention on the process of second-harmonic (SH) generation. Excitation of localized surface plasmon polaritons at the fundamental frequency in these structures can remarkably modify the response of the system by enhancing surface and/or bulk nonlinear contributions to the overall SH field. Moreover, the radiation pattern of the generated field can be controlled and directed. Indeed, the overall structure behaves as a nonlinear nanoantenna for the SH harmonic field, whose emission pattern can be tailored.

In the last few years, thanks to the development of nanotechnologies, it has become possible to manipulate matter on the nanoscale and to create artificial materials with tailored unconventional properties with respect to those of bulk materials. ${ }^{1}$ The possibility to achieve light confinement at subwavelength scales has been demonstrated in metal nanostructures supporting localized plasmon resonances, i.e., the collective resonant oscillation of electrons excited by light of appropriate frequency. Thus, the optical response of metal nanoparticles and metal nanostructures has greatly increased.

Our activity is mostly devoted to studying the nonlinear response of metal nanoparticles. The main concept that has been exploited is based on the possibility of achieving high-field confinement in coupled nanoresonators. Coupling is, in turn, responsible for the formation of resonant modes that can be localized on small portions of the structure or distributed over the whole structure. ${ }^{2}$ Different field profiles can be obtained by varying the parameters of the input field (for example, the frequency or the spatial phase profile). Zones of high-field confinement, called hot spots, are characterized by local field intensity values of three or four orders of magnitude higher than the incident field's intensity. ${ }^{3}$ Thus, considering the process of second harmonic generation (SHG), the emission comes from the hot spots with an enhancement of eight orders of magnitude with respect to the portions of the structure where the field is not localized. By controlling the hot spot position, the radiation pattern of the generated field can be controlled and directed the same way as it would happen by engineering a feeder in a nanoantenna.

Recently, experimental techniques have been developed to map the hot spots and to study the connection between field localization and SHG properties in optical metamaterials. ${ }^{3,4}$ Since the local field distribution in nanoplasmonic structures is highly inhomogeneous, it is not obvious to establish the role of surface and bulk nonlinearities as well as their relative weights in the overall second harmonic signal. This subject has been investigated, both theoretically and experimentally, ${ }^{5-7}$ but the relevance of bulk and surface nonlinearities in nanoplasmonic structures is still under debate. Our calculations have been performed by extending to three-dimensional (3-D) structures an algorithm based on the Green's tensor method, previously developed for calculation of SHG in two-dimensional structures. ${ }^{8,9}$ With the aim to provide a tool for separating experimental investigation of nonlinear surface and bulk contributions, we studied resonant dipole gold nanoantennas. The SHG as a function of the wire's cross-section size was investigated in both the near- and far- field regimes, revealing that different geometries and input field polarizations lead to different emission patterns of the second harmonic field. We calculated the second harmonic nonlinear differential scattering cross-section of a $13 \times 13-\mathrm{nm}^{2}$ antenna when a fundamental frequency field with a wavelength of $800 \mathrm{~nm}$ is considered either 


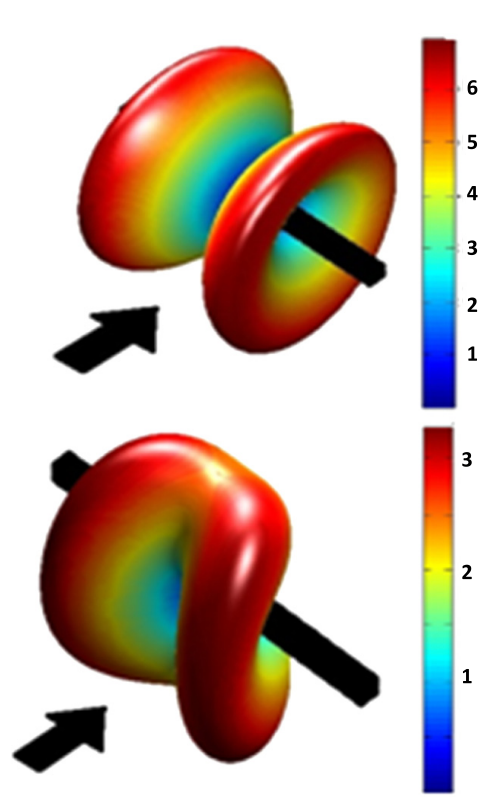

(a)

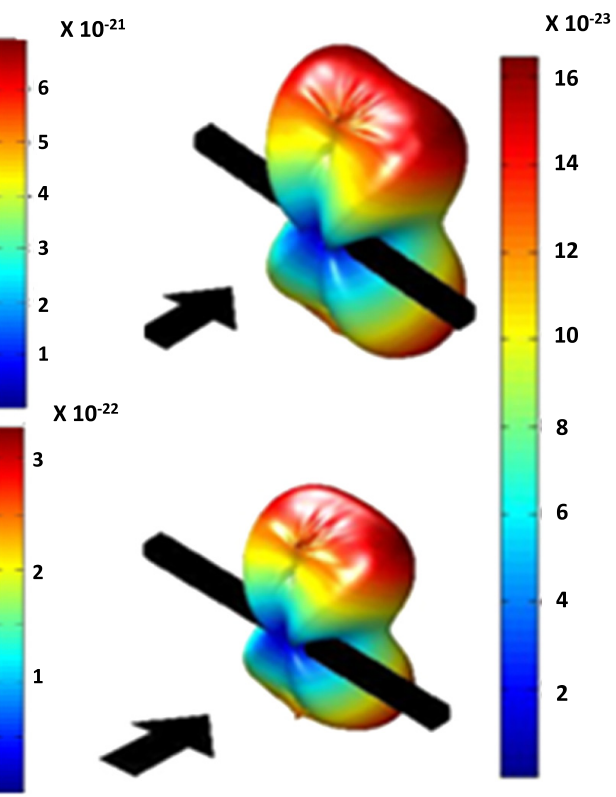

(b)

Fig. 1 Second harmonic differential scattering cross section $\left(\mathrm{cm}^{2} / \mathrm{W}\right)$ for (a) TE and (b) TM polarization modes calculated by taking into account (upper images) and neglecting (lower images) the nonlinear surface contributions. Black arrows indicate direction of propagation of the pump field; the nanoantenna has been interposed only as a reference for the 3-D scattering distribution.

transverse-electric (TE) or transverse-magnetic (TM) polarized. Our calculations show that there are two different behaviors for the TE and TM pump polarization. Indeed when a TM-polarized pump is considered, bulk terms dominate over surface terms by over one order of magnitude. With a good level of accuracy, the signal generated can be considered as related only to the bulk terms. On the other hand, when surface terms are comparable and/or bigger than bulk contribution (i.e., for TE-polarization, in the considered scheme) the calculated far-field pattern can be very different if both terms are considered with respect to the case where surface terms are neglected. As shown in Fig. 1, the difference is not only in the value of the differential nonlinear scattering cross-section but also in the angular distribution of the generated second harmonic field. By comparing the two plots we can state that the forward generated second harmonic, with respect to the direction of the pump, is related only to the surface terms. These results suggest a way to perform an indirect measurement of the nonlinear coefficient for the bulk and for the surface terms separately.

Control of the nonlinear emission can be further achieved by considering coupled systems composed by a small ensemble of nanoresonators. We adapted our numerical model to investigate second harmonic generation in coupled $\mathrm{Ag}$ rods. The building block of the considered system are silver rods of rectangular section (length $=212 \mathrm{~nm}$, thickness $=38 \mathrm{~nm}$ ) separated by a $28-\mathrm{nm}$ gap. With these values of the geometrical parameters the system has a resonant behavior in the wavelength range around $1 \mu \mathrm{m}$. Complex structures provide a richer variety of cases. For example, we considered a structure composed of eight rods. In Fig. 2(a) and 2(b), we show that the second harmonic main emission angle can be controlled on a range of 20 deg by tuning the pump field wavelength from 920 to $960 \mathrm{~nm}$. A similar behavior can be obtained by controlling the fundamental field hot spots with two counter-propagating beams. Indeed, in this case the position of the hot spots depends on the relative phase between the two coherent counter-propagating beams. ${ }^{10}$

As a perspective for future work, we are implementing plasmonic nanoresonators in dielectric waveguide systems as sketched in Fig. 2(c), to explore the potential applications of plasmonic nanostructures for integrated and controllable sources. The pump field will be injected in the dielectric waveguide and a second pump beam can be injected in the opposite direction in order to control the localization pattern of the hot spots. A second harmonic field will then be emitted in a small spatial region. Optimization of the structures in order to obtain high 


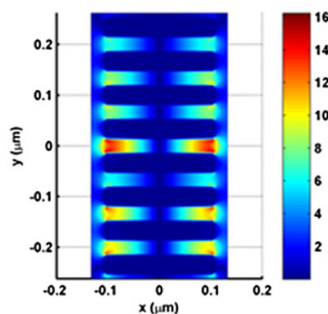

(a)

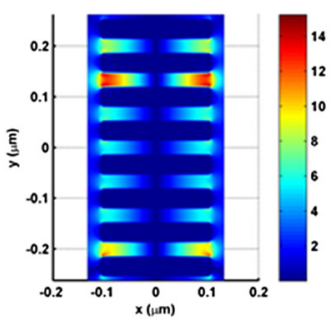

(c)

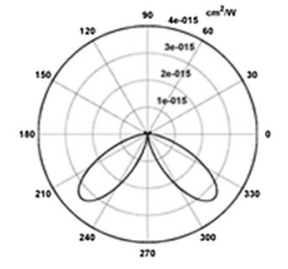

(b)

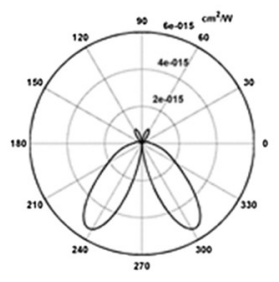

(d)
GUIDED EXCITATION

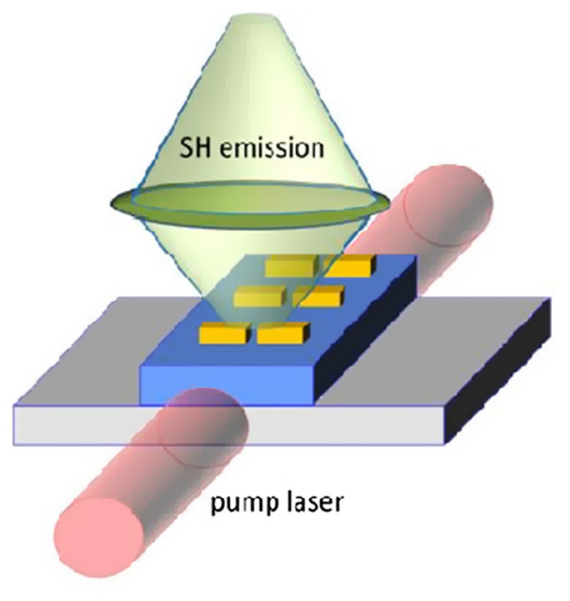

(e)

Fig. 2 Modulus of the pump field normalized with respect to the amplitude of the $x$ polarized, upward propagating, incident field with a wavelength of (a) $920 \mathrm{~nm}$ and (c) $960 \mathrm{~nm}$. Differential nonlinear scattering cross-section for the second harmonic field wavelength of (b) $460 \mathrm{~nm}$ and (d) $480 \mathrm{~nm}$. (e) Sketch of the proposed scheme for further work.

directionality or high selectivity can be performed by changing the size and shapes of the nanoresonators and it could be possible to study the fluorescence of a single molecule ${ }^{11}$ located in the hot spot by far-field detection at the proper angle of emission.

\section{Acknowledgments}

We thank M. Bertolotti and C. Sibilia for useful discussions and fruitful collaboration.

\section{References}

1. N. Liu et al., "Three-dimensional photonic metamaterials at optical frequencies," Nat. Mater. 7(1), 31-37 (2007), http://dx.doi.org/10.1038/nmat2072.

2. M. Centini et al., "Coupled 2D Ag nano-resonator chains for enhanced and spatially tailored second harmonic generation," Opt. Express 19(9), 8218-8232 (2011), http://dx.doi.org/ 10.1364/OE.19.008218.

3. V. K. Valev et al., "Plasmon-enhanced sub-wavelength laser ablation: plasmonic nanojets," Adv. Mater. 24(10), OP29-OP35 (2012), http://dx.doi.org/10.1002/adma.201103807.

4. J. Butet et al., "Optical second harmonic generation of single metallic nanoparticles embedded in a homogeneous medium," Nano Lett. 10(5), 1717-1721, (2010), http://dx .doi.org/10.1021/nl1000949.

5. A. Benedetti et al., "Second harmonic generation from 3D nanoantennas: on the surface and bulk contributions by far-field pattern analysis," Opt. Express 19(27), 26752-26767 (2011), http://dx.doi.org/10.1364/OE.19.026752.

6. Y. Zeng and J. V. Moloney, "Volume electric dipole origin of second-harmonic generation from metallic membrane with noncentrosymmetric patterns," Opt. Lett. 34(18), 2844-2846 (2009), http://dx.doi.org/10.1364/OL.34.002844.

7. F. X. Wang et al., "Surface and bulk contributions to the second-order nonlinear optical response of a gold film," Phys. Rev. B 80(23), 233402 (2009), http://dx.doi.org/10 .1103/PhysRevB.80.233402.

8. A. Benedetti et al., "Engineering the second harmonic generation pattern from coupled gold nanowires," J. Opt. Soc. Am. B 27(3), 408-416 (2010), http://dx.doi.org/10.1364/JOSAB .27 .000408 . 
9. M. Centini et al., "Second harmonic generation in plasmonic nanoresonators," Proc. SPIE 8423, 84231I (2012), http://dx.doi.org/10.1117/12.923214.

10. G. Volpe et al., "Controlling the optical near field of nanoantennas with spatial phaseshaped beams," Nano Lett. 9(10), 3608-3611 (2009), http://dx.doi.org/10.1021/n1901821s.

11. A. Kinkhabwala et al., "Single-molecule fluorescence enhancements produced by a Bowtie nanoantenna," Nat. Photon. 3(11), 654-657 (2009), http://dx.doi.org/10.1038/nphoton .2009 .187 . 